\title{
A Robustness Comparison of Two Algorithms Used for EEG Spike Detection
}

\author{
Sahbi Chaibi ${ }^{1, *}$, Tarek Lajnef ${ }^{1}$, Abdelbacet Ghrob ${ }^{1}$, Mounir Samet ${ }^{1}$ and Abdennaceur Kachouri ${ }^{1,2}$

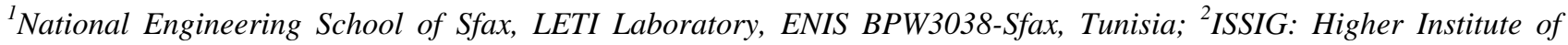 \\ Industrial Systems, Gabes CP 6011, Tunisia
}

\begin{abstract}
Spikes and sharp waves recorded on scalp EEG may play an important role in identifying the epileptogenic network as well as in understanding the central nervous system. Therefore, several automatic and semi-automatic methods have been implemented to detect these two neural transients. A consistent gold standard associated with a high degree of agreement among neuroscientists is required to measure relevant performance of different methods. In fact, scalp EEG data can often be corrupted by a set of artifacts and are not always served as data of gold standard. For this reason, the use of intracerebral EEG data mixed with gaussian noise seems to best resemble the output of scalp EEG brain and serves as a consistent gold standard. In the present framework, we test the robustness of two important methods that have been previously used for the automatic detection of epileptiform transients (spikes and sharp waves). These methods are based respectively on Discrete Wavelet Transform (DWT) and Continuous Wavelet Transform (CWT). Our purpose is to elaborate a comparative study in terms of sensitivity and selectivity changes via the decrease of Signal to Noise Ratio (SNR), which is ranged from $10 \mathrm{~dB}$ up to $-10 \mathrm{~dB}$. The results demonstrate that, DWT approach turns to be more stable in terms of sensitivity, and it successfully follows the detection of relevant spikes with the decrease of SNR. However, CWT-based approach remains more stable in terms of selectivity, so that, it performs well in terms of rejecting false spikes compared to DWT approach.
\end{abstract}

Keywords: CWT, DWT, Epilepsy, noisy neural data, Stereo-Electroencephalography (SEEG).

\section{INTRODUCTION}

Studying and monitoring the neural activities of the human brain has become one of the most interesting and challenging fields of biomedical engineering [1]. Identification of the specific structural brain abnormalities in nervous signals is one of the main goals of neuroscientists and biomedical engineers since it provides valuable information about the current and future health status of epileptic patients. In addition, neural epileptic features may play an important role in understanding the central nervous system. Actually, interictal discharges like spikes and sharp waves [2-5] are potentially serving as distinctive biomarkers of epileptogenic brain. The International Federation of Societies for Electroencephalography and Clinical Neurophysiology (IFSECN) $[2,6]$ defines a spike as a transient, clearly distinguished from background activity, with duration of $20 \mathrm{~ms}$ up to 70 ms. Sharp wave- same as spike, but with duration ranged from $70 \mathrm{~ms}$ up to $200 \mathrm{~ms}$.

Over the last years, several methods $[1,2,6]$ have been proposed for automatic detection of neural spikes recorded on scalp EEG. In order to evaluate the accuracy and efficiency of the proposed methods, a consistent gold standard (ground truth neural spikes) associated with a high degree of agreement among neuroscientists are required. As a matter of fact, scalp EEG data can often be contaminated or corrupted by a set of artifacts like sleep transients, eye blink, eye

*Address correspondence to this author at the National Engineering School of Sfax, LETI Laboratory, ENIS BPW3038-Sfax, Tunisia;

Tel: +216 25621 084; E-mail: sahbi.chaibi@yahoo.fr movement, muscle, ECG patterns and electrode artifacts [6]. Thus, the visual marking of neuronal spikes buried in these types of noise and interferences is believed to be a complicated issue. Although neuroscientist experts are skillful, false identification due to subjective analysis and disagreement between them for the same record can easily occur, especially in the case of scalp EEG signals with low SNR. Currently, the major problem is that the use of scalp EEG data could represent a lack of a consistent gold standard, which can affect the evaluation of relevant performance for different detection methods.

To tackle this problem, other methodological challenges have been proposed [1, 7-9]. In fact, the use of mixed intracerebral EEG data with random noise seems to be highly accurate and serve as a reliable gold standard used in the assessment of the performance of different detectors. These types of data best resemble and are highly correlated with the output scalp EEG of the brain. In this study, we have two main objectives: our first goal is to evaluate the robustness degree in terms of sensitivity and selectivity changes via the decrease of SNR. Mainly, we have used two approaches that have been previously used for the automatic detection of epileptiform spikes, based respectively on: Discrete Wavelet Transform (DWT) [6, 10] and Continuous Wavelet Transform (CWT) [3, 11]. The robustness degree was evaluated via an epileptic SEEG data mixed with different noise levels range from $10 \mathrm{~dB}$ up to $-10 \mathrm{~dB}$. Our second purpose is to elaborate an overall comparison between the two approaches.

The structure of the paper is ordered as follows: Section 1 presents the Introduction. Section 2 describes the materials and methods. Section 3 concerns our results and discussion. 
The last section presents the conclusion, and also the directions for future research.

\section{MATERIALS AND METHODS}

\subsection{Intracerebral EEG Data \& Preprocessing}

The EEG data used in this study was recorded by semirigid multi-lead electrodes that were stereotactically implanted in the patient's brain. The stereotactic-EEG (SEEG) electrodes have a diameter of $0.8 \mathrm{~mm}$ and generally consist of 10 to 15 contact leads, $2 \mathrm{~mm}$ wide and $1.5 \mathrm{~mm}$ apart (DIXI Medical, Besançon, France). The intracerebral recordings were conducted at the Lyon Neurological Hospital (Lyon, France) using a video-SEEG monitoring system (Micromed, Treviso, Italy), which allowed the simultaneous data recording from up to 128 depth-EEG electrode sites. The data were sampled at $256 \mathrm{~Hz}$. At the time of acquisition the data is recorded using a reference electrode located in white matter, each electrode trace is re-referenced with respect to its direct neighbor (bipolar derivations). This bipolar montage helps eliminate signal artifacts common to adjacent electrode contacts (such as the $50 \mathrm{~Hz}$ power supply artifact) and achieves a high local specificity by canceling out effects of distant sources that are equally spread to both adjacent sites through volume conduction. The spatial resolution achieved by the bipolar SEEG is on the order of $3 \mathrm{~mm}$.

Our methods were tested on 2-minute segments of SEEG data containing only epileptic spikes. Visual marking of spikes was performed by one expert trained in electrophysiology and spikes analysis. All the marked events were immediately saved numerically in a database. That would serve subsequently as a gold standard for the evaluation and the measurement of the performance detection. Then, the chosen data were mixed with the Gaussian noise using six SNR values $(10 \mathrm{~dB}, 3 \mathrm{~dB}, 0 \mathrm{~dB},-3 \mathrm{~dB}$ and $-10 \mathrm{~dB})$, associated with 10 trials for each SNR. The variation of SNR values in human scalp EEG is comprised approximately between $10 \mathrm{~dB}$ and $-10 \mathrm{~dB}[12,13]$. Therefore, only this range was taken into consideration in our study. Subsequently, the obtained data were approximated as scalp EEG recordings and considered as a consistent gold-standard data set. The gold standard will be needed to test the stability of various test methods against the noise. The visual marking of epileptic spikes was performed via the signal visualization tools available in the EEGLAB [14] and ELAN [15] toolboxes. The processing and analysis of all signals were performed with custom codes which were implemented using MATLAB 2012a software.

\subsection{Discrete Wavelet Transform (DWT)}

The basic idea of DWT wavelet analysis consists in expressing a signal $(s)$ as a linear combination of a particular set of functions, obtained by dilating and shifting a mother wavelet. The decomposition of the signal leads to a set of coefficients $(C)_{n . k}$ called wavelet coefficients based on the following equation:

$(C)_{n . k}=<s, \Psi_{n, k}>=\int s(x) \Psi_{n, k}(x) d x$

Where $\Psi_{n, k}(x)=a^{-\frac{n}{2}} \Psi\left(a^{-n} x-k b\right)$, where $\mathrm{a}=2$ and $\mathrm{b}=1$.
The original signal can be expressed as a linear combination of the wavelet functions weighted by the wavelet coefficients. The wavelet transformation analyses the signal at different frequency bands, with different resolutions by decomposing the signal into a coarse approximation and detail information. The selection of suitable wavelet and the number of decomposition levels is very important in the analysis of signals using DWT wavelet transformation. The Daubechies wavelet $(\mathrm{Db} 4)$ has been previously chosen for automatic EEG spike detection [6,10], because it yields the highest correlation coefficients with the epileptic spike among the wavelet bases available in the MATLAB Toolbox and it best matches the shape and frequency characteristics of epileptic spikes $[6,10]$. On the other hand, the appropriate band ranging from $4 \mathrm{~Hz}$ up to $16 \mathrm{~Hz}$ has been selected to detect epileptic spikes [6]. In fact, this band is very specific and not affected by different types of artifacts and nonepileptic transients like sleep transients, eye blink, eye movement, muscle, ECG patterns and electrode artifacts and interferences [6]. The number of decomposition levels was chosen in terms of these frequency components. In the present study, the sampling frequency is $256 \mathrm{~Hz}$, since the EEG band taken into consideration is comprised between 4 and 16 $\mathrm{Hz}$, so the number of decomposition levels was chosen to be 5 . Thus, the input signal is decomposed into five details subsignals and one final approximation: D1 $(64-128 \mathrm{~Hz}), \mathrm{D} 2$ $(32-64 \mathrm{~Hz}), \mathrm{D} 3(16-32 \mathrm{~Hz}), \mathrm{D} 4(8-16 \mathrm{~Hz}), \mathrm{D} 5(4-8 \mathrm{~Hz})$ and A5 $(0-4 \mathrm{~Hz})$. After that, the two sub-signals (D5 and D4) are summed up across all time samples. Subsequently, the obtained signal is rectified and smoothened. The spikes are detected when the value of the obtained final signal is greater than a threshold. The threshold is defined as the sum of the mean and the standard deviation of the obtained final signal. The following Data flow diagram sums up the main steps of DWT-based approach (Fig. 1).

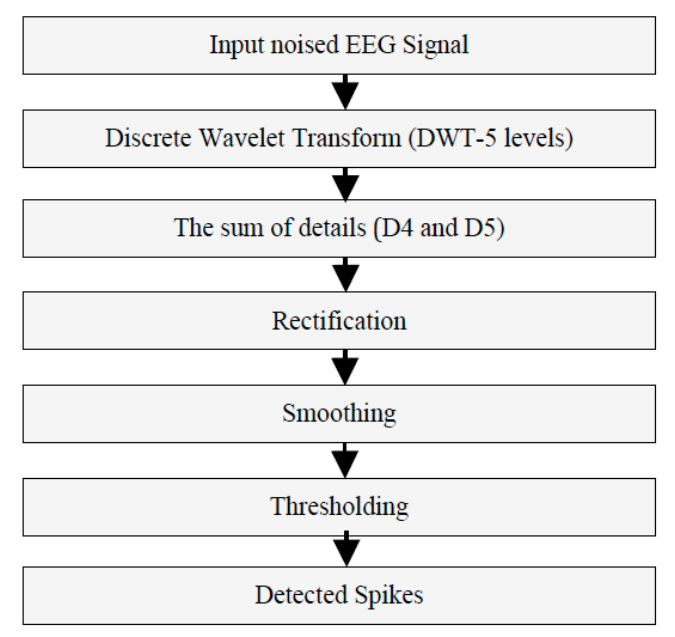

Fig. (1). Data flow diagram of the DWT method.

\subsection{Continuous Wavelet Transform (CWT)}

Continuous Wavelet Transform (CWT) coefficients are computed by continuous scaling and translating. This algorithm is inspired from the methods proposed by [3, 12], but it is very similar to DWT method in its implementation. The continuous wavelet transform (CWT) is defined as the corre- 
lation between the signal to be analyzed and the wavelet functions. The used wavelet $\psi(t)$ in our case is defined as follows:

$\psi(t)=\frac{1}{\sqrt{2 \pi \cdot \sigma_{t}^{2}}} e^{i 2 \pi f_{c} t} e^{\frac{-t^{2}}{2 \cdot \sigma_{t}^{2}}}$

Where $f_{c}$ is the characteristic frequency of the mother wavelet, the standard deviation of the Gaussian window in equation (2) is set to $\sigma_{t}=1$. Selection of the proper wavelet and the number of scales is very important for signal analysis using the CWT. Hence, in order to have a high correlation with epileptic spikes, the wavelet family was chosen in such a way that the ratio frequency to bandwidth was equal to $f_{c} / \sigma_{f}=3$, where $\sigma_{f}=1 / 2 \pi . \sigma_{t}$. Approximately the same band was used in this method like DWT, which ranged from $4 \mathrm{~Hz}$ up to $16 \mathrm{~Hz}$ with a step of $1 \mathrm{~Hz}$. The mother let $\psi(t)$, defined above, can be scaled by a factor $a$ in frequency and translated by an amount $b$ in time as follows:

$\psi(a, b)=\psi\left(\frac{t-b}{a}\right)$

$\psi(a, b)$ is known as a daughter wavelet. Scale $a$ is related to a pseudo-frequency $f$ according to the following relationship:

$a=\frac{f_{c}}{(\mathrm{~T} . f)}$

Where $\mathrm{T}$ is the sampling period and $f_{c}$ is equal to 0.47 $\mathrm{Hz}$. The wavelet power $w(a, b)$ is then computed as follows:

$w(a, b)=\left|\frac{1}{\sqrt{a}} \int_{-\infty}^{+\infty} f(x) \overline{\psi\left(\frac{x-b}{a}\right)} d x\right|^{2}$

Time frequency map $\mathrm{X}(\mathrm{f}, \mathrm{t})$ is computed from wavelet power $w(a, b)$ by transforming scale $a$ into pseudofrequency $\mathrm{f}$ using equation (4) and replacing $\mathrm{b}$ with time t. Then, for each time sample, the average of all coefficients associated with different frequencies is done. Subsequently, the obtained signal is smoothed before selecting the threshold value. The detection threshold is defined as the sum of the mean and the standard deviation of the obtained final signal. Finally, detected spikes are positioned in time depending on the signal of interest. The following Data flow diagram sums up the main steps of CWT-based approach (Fig. 2).

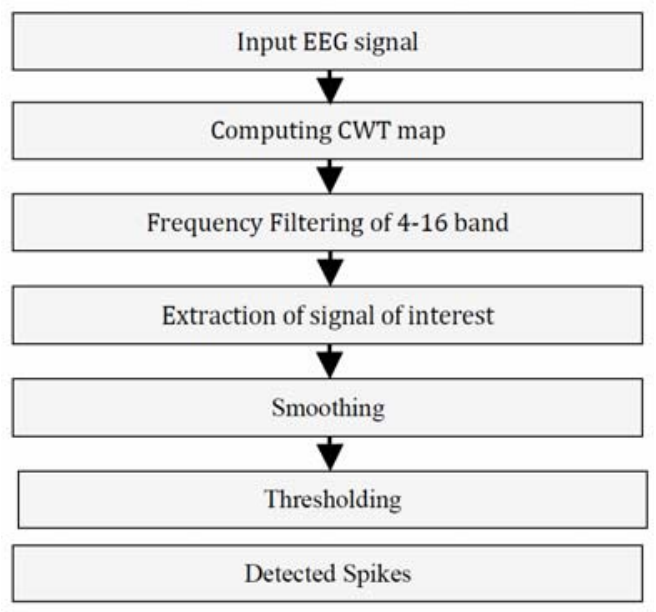

Fig. (2). Data flow diagram of the CWT method.

\subsection{Performance Metrics}

In this section, we present a brief review of concepts related to the performance's evaluation. Assuming both spike also termed as gold-standard in EEG are properly annotated by an experienced neuroscientist. By comparing the output of the automatic detection procedure to visual identification by an expert, we derived the total true positive (TP) of spikes and False positive (FP). The performance of our tested approaches was evaluated only in terms of sensitivity and selectivity. Indeed, sensitivity is defined as the capability of the system to detect correctly relevant spikes. However, selectivity is defined as the capability of the system to select precisely true spikes and reject the spurious spikes correctly.

$$
\begin{aligned}
& \text { Sensitivity }=\frac{\mathrm{TP}}{\mathrm{TP}+\mathrm{FN}} * 100 \% \\
& \text { Selectivity }=\frac{\mathrm{TP}}{\mathrm{TP}+\mathrm{FP}} * 100 \%
\end{aligned}
$$

The specificity and the system accuracy were biased in this study because the tested EEG segments contain only epileptic spikes. TP is defined as the number of spikes visually marked by the reviewer and correctly detected by the automatic detectors. FN is defined as the number of spikes visually marked by the reviewer and missed by the automatic detectors. FP is defined as the number of spurious spikes misclassified as true spikes by the automatic detectors.

\section{RESULTS AND DISCUSSION}

Scalp EEG are generally contaminated by a set of artifacts and noises, which makes the discrimination between epileptic spikes and non-pathological transients in some cases is less obvious. However, spikes recorded on intracerebral EEG can be easily distinguished by visual inspection and are associated with a perceived ground truth. In fact, the use of mixed intracerebral EEG with noise instead using directly scalp EEG is very useful. This methodology reflects a valuable and practical procedure that can be used to estimate accurately the relevant performance on the one hand, and to test the stability of such developed algorithm against noise by following its SNR variation on the other hand. In this study, we compared the stability of different approaches across random Gaussian noise. Our results of automatic spike detection with different SNR and trials are illustrated in Fig. (3) and Table $\mathbf{1}$

Our main findings are summarized as follows: different methods were approximately stable in low SNR (10 and 3 $\mathrm{dB}$ ) in which a high sensitivity and selectivity were obtained compared to the result without noise. For $\mathrm{SNR}=0,-3$ and $-10 \mathrm{~dB}$, DWT remains more stable in terms of sensitivity compared to CWT with the decrease of SNR. However, CWT based approach remains more stable in terms of selectivity compared to DWT approach, so that, it performs well in terms of the rejection of false spikes, which come essentially from noise. The average sensitivity and the average selectivity achieved by DWT approach via all SNR are respectively $96.88 \%$ and $63.46 \%$. On the other hand, the average sensitivity and the average selectivity achieved by CWT approach via all SNR are respectively: $93.01 \%$ and $79.10 \%$. Our simulations have been carried out using a DELL-PC with Intel(R) Core (TM) i3 CPUM2350 2.30GHz and 4-GB RAM by MATLABR2012. The CPU times were assessed 
Table 1. Performance of spikes detection using DWT and CWT based-approaches with different SNR and different trials.

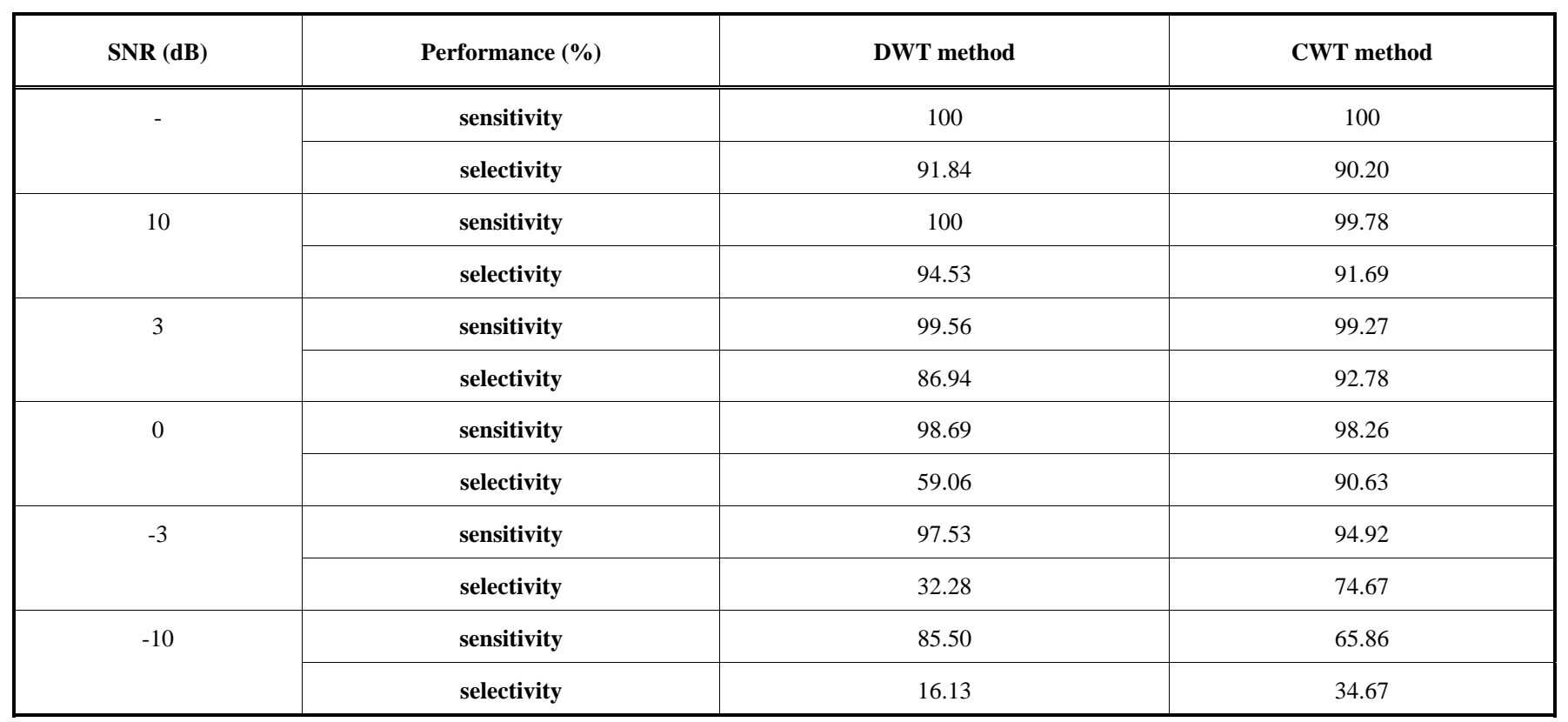
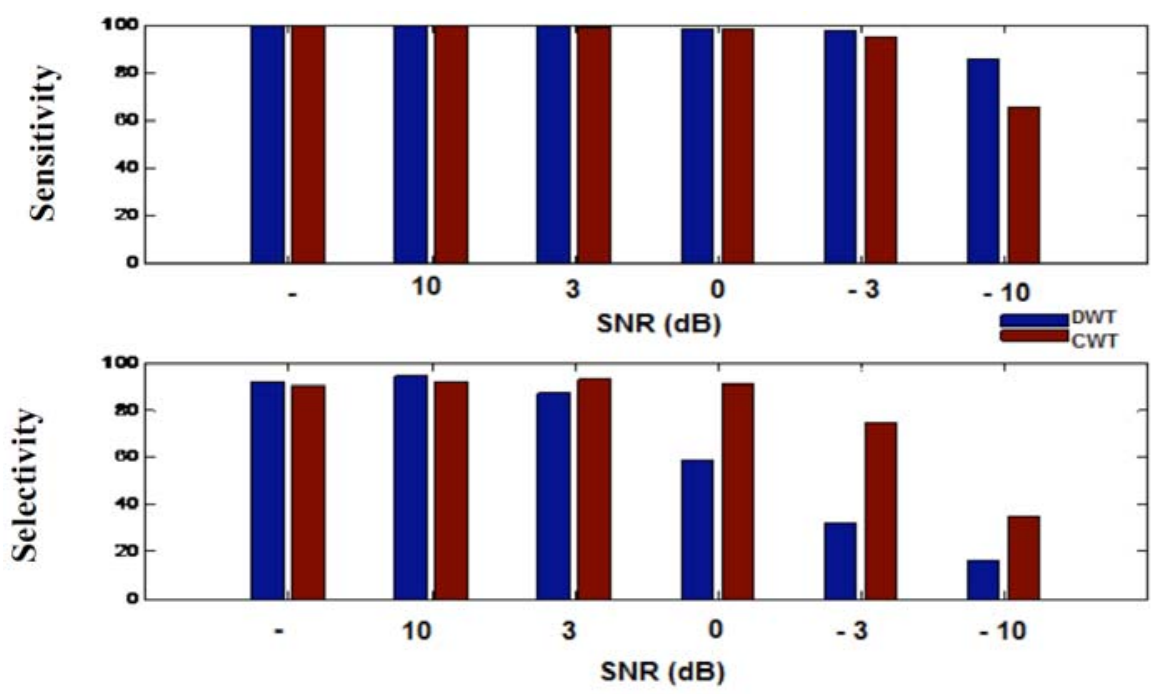

Fig. (3). Graph of performance of different methods and different SNR.

for one minute EEG data; it is $5.473 \mathrm{~s}$ for DWT approach and $5.117 \mathrm{~s}$ for CWT method. Overall, CWT based method seems to be more robust than DWT method in terms of performance.

In the current paragraph, we aim to provide some examples of spikes detection using respectively DWT approach and CWT approach. The left side of Fig. (4) presents an example of spike detection based on the DWT method. Fig. (4a) gives an illustration of signal SEEG with spikes. Fig. (4b) shows the result of the original SEEG data mixed with the random Gaussian noise $(\mathrm{SNR}=-3 \mathrm{~dB})$. Fig. (4c) shows the result of summation, rectification and smoothing of details D4 and D5. The bottom plot (Fig. 4d) shows the position of detected spikes.

The right side of Fig. (4) presents, an example of spikes detection based on the CWT method. Fig. (4e) gives an illus- tration of signal SEEG with spikes. Fig. (4f) shows the result of the original SEEG data mixed with the random Gaussian noise $(\mathrm{SNR}=-3 \mathrm{~dB})$. Fig. (4g) shows the map CWT and its thresholding. The bottom plot (Fig. 4h) shows the detected spikes using CWT.

It may be much more interesting and challengeable to compare our methods with different other algorithms. However, the comparison between spikes detectors is not an easy task. The following reasons can be considered as difficulties in assessing or comparing the performances of automatic spikes detection methods: varying EEG recording techniques, different electrode locations and pathologies across patients, different definitions for spikes (i.e. frequency bands), and lack of gold standard for defining spikes events. 
a)-Clinical epileptic SEEG data.
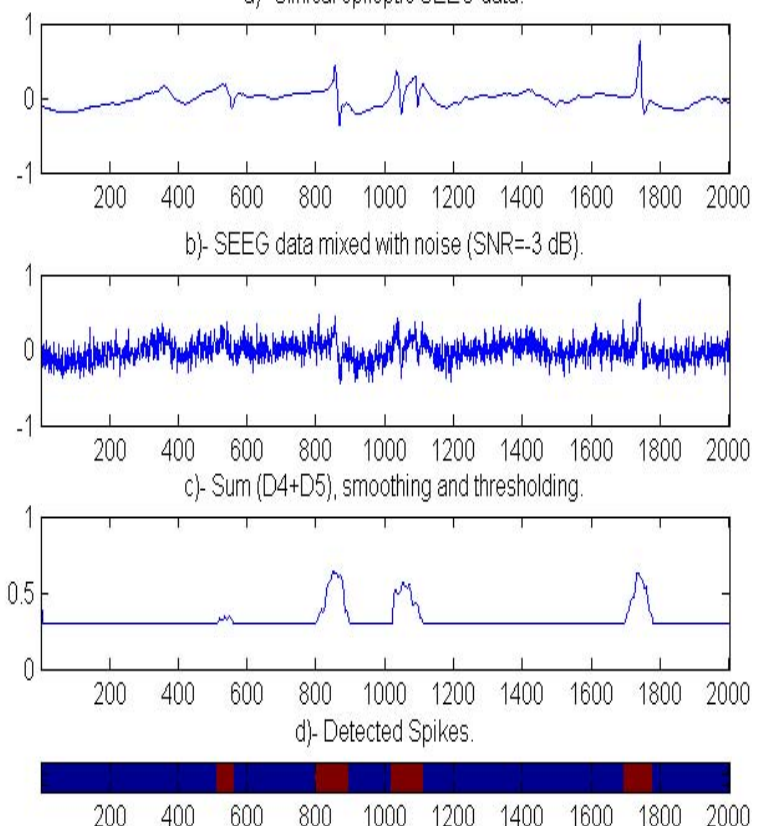

e)-Clinical epileptic SEEG data.
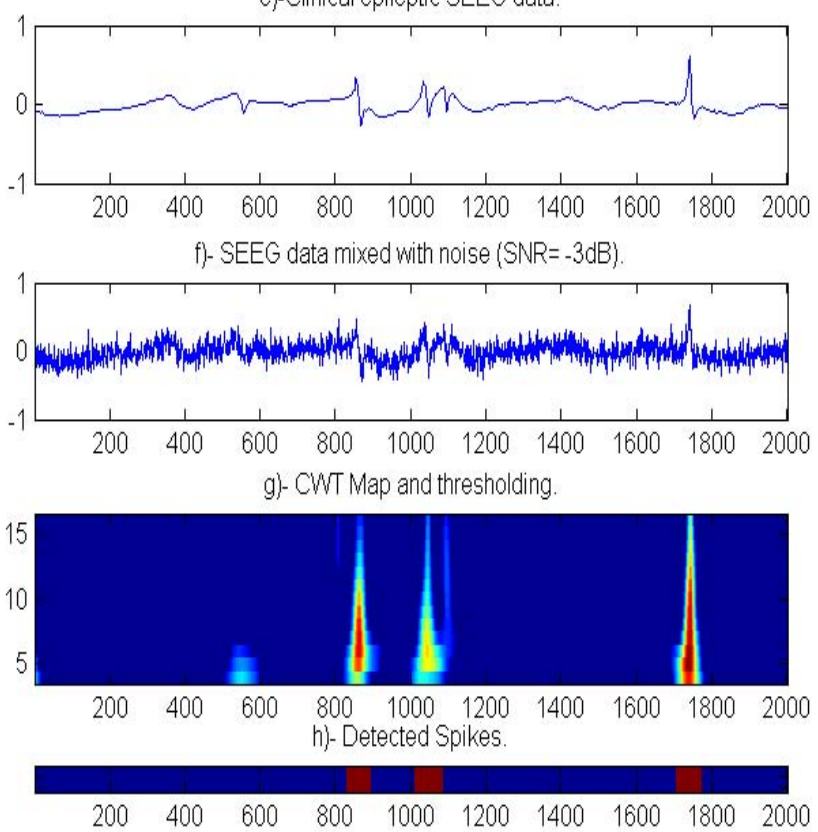

Fig. (4). Example of spikes detection based respectively on DWT and CWT.

\section{CONCLUSION}

Visual inspection and identification of spikes in epilepsy recordings are a time-consuming and a tedious procedure especially in the case of scalp EEG. So a sensitive selective, specific, accurate and robust technique of epileptic spikes detection is an important and useful tool that can assist neuroscientists and biomedical engineers. Different methods described in this paper can work well in high SNR. However, with the decrease of SNR, DWT based approach can perform well than CWT approach in terms of sensitivity. Yet, CWT-based approach remains more stable in terms of selectivity. As a final finding, the conjunction of the two methods can work well for the detection of epileptic spikes and sharp waves. Still, extensive testing and validation on more data sets are required. Also, further investigations are needed to quantify other performance measures such as specificity, and system accuracy using a complete set of data including both epileptic spikes and non-pathological transient events. It is also very interesting to compare our two methods with other different methods using the same methodology.

\section{CONFLICT OF INTEREST}

The authors confirm that this article content has no conflict of interest.

\section{ACKNOWLEDGEMENTS}

Authors wish to thank the Lyon Neurological Hospital (Lyon, France) for kindly providing database which we used to test and compare the performance of different methods described in this study. The authors would like also to thank
PhD Thouraya Ezzine from the Faculty of Arts and Human Sciences of Sfax for her help with English.

\section{PATIENT'S CONSENT}

Declared none.

\section{REFERENCES}

[1] M.H. Zarifia, N.K. Ghalehjogh, and M. Baradaran-nia, "A new evolutionary approach for neural spike detection based on genetic algorithm", Expert Syst. Appl., vol. 42, pp. 462-467, 2015.

[2] F.D. de Moraes, and D.A. Callegari, Automated Detection of Interictal Spikes in EEG: A Literature Review, Pontifícia Universidade Católica do Rio Grande do Sul Av. Ipiranga, 6681-Porto Alegre/RS, Brasil, 2014.

[3] P.K. Das, A. Mohapatra, and M.R. Panda, "Epilepsy disorder detection from EEG signal", Int. J. Intell. Comput. Appl. Sci., vol. 1, no. 1 , pp. 41-49, 2013 .

[4] S. Chaibi, T. Lajnef, Z. Sakka, M. Samet, and A. Kachouri, "A reliable approach to distinguish between transient with and without HFOs using TQWT and MCA", J. Neurosci. Methods, vol. 232, pp. 36-46, 2014 .

[5] S. Chaibi, Z. Sakka, T. Lajnef, M. Samet, and A. Kachouri, "Automated detection and classification of high frequency oscillations (HFOs) in human intracereberal EEG", Biomed. Signal Process. Control, vol. 8, pp. 927-934, 2013.

[6] K.P. Indiradevi, E. Elias, P.S. Sathidevi, S.D. Nayak, and K. Radhakrishnan, "A multi-level wavelet approach for automatic detection of epileptic spikes in the electroencephalogram", Comput. Biol. Med., vol. 38, pp. 805-816, 2008.

[7] H. Azami, J. Escudero, A. Darzi, and S. Sanei, "Extracellular spike detection from multiple electrode array using novel intelligent filter and ensemble fuzzy decision making", J. Neurosci. Methods, vol. 239, pp. 129-138, 2015.

[8] H. Azami, and S. Sanei, "Spike detection approaches for noisy neuronal data; assessment and comparison", Neurocomputing., vol. 133, pp. 491-506, 2014. 
[9] H. Azami, M. Saraf, K. Mohammadi, and S. Sanei, "A novel extracellular spike detection approach for noisy neuronal data", Artif. Intell. Signal Process., vol. 427, pp. 332-343, 2014.

[10] L. Guo, D. Rivero, J. Dorado, J.R. Rabunal, and A. Pazos, "Automatic epileptic seizure detection in EEGs based on line length feature and artificial neural networks", J. Neurosci. Methods, vol. 191, pp. 101-109, 2010.

[11] M. Latka, Z. Was, A. Kozik, and B. West, "Wavelet analysis of epileptic spikes," Phys. Rev. E Stat. Nonlin. Soft Matter Phys., vol. 67, pp. 105-122, 2003.

[12] D.M. Goldenholz, S.P. Ahlfors, M.S. Hämäläinen, D. Sharon, M. Ishitobi, L.M. Vaina, and S.M. Stufflebeam, "Mapping the signalto-noise-ratios of cortical sources in magnetoencephalography and electroencephalography", Hum. Brain Mapp., vol. 30, pp. 10771086, 2009.

[13] A. Kachenoura, D. Safieddine, A. Albera, G. Birot, F. Wendling, L. Senhadji, and I. Merlet, "Blind source separation methods applied to muscle artefacts removing from epileptic eeg recording: a comparative study", In: Proceedings of RITS (Colloque National Recherche en Imagerie et Technologies pour la Santé), Rennes, France, 2011.

[14] http://www.sccn.ucsd.edu/eeglab

[15] P.E. Aguera, K. Jerbi, A. Caclin, and O. Bertrand, " ELAN: A software package for analysis and visualization of MEG, EEG, and LFP signals", Comput. Intell. Neurosci., vol. 2011, p. 158970 , 2011.

Received: January 02, 2015

Revised: May 31, 2015

Accepted: June 02, 2015

() Chaibi et al.; Licensee Bentham Open.

This is an open access article licensed under the terms of the Creative Commons Attribution Non-Commercial License (http://creativecommons.org/licenses/by-nc/3.0/) which permits unrestricted, non-commercial use, distribution and reproduction in any medium, provided the work is properly cited. 